Ann. Génét. Sél anim., I97o. 2 (I), 85-99.

\title{
GÈNES INFLUANT SUR LA VARIANCE PHÉNOTYPIQUE DE L'ÂGE AU PREMIER GEUF ET LA COMPOSITION DE CETTE VARIANCE CHEZ LA POULE DOMESTIQUE
}

\author{
P. MÉRAT \\ avec la collaboration technique de L. Durand et A. Bordas \\ Station centrale de Génétique animale, \\ Centre national de Recherches zootechniques, 78-Jouy-en-Josas \\ Institut national de la Recherche agronomique
}

\section{RÉSUMÉ}

Pour l'âge à la ponte du premier œuf des poulettes, une différence hautement significative de la variance a été trouvée, entre hétérozygotes et homozygotes récessifs, à trois loci : E (extension/restriction du noir dans le plumage), $\mathrm{C}$ (présence /absence de mélanines dans le plumage), $\mathrm{R}$ (crête en rose/simple). Les hétérozygotes pour le noir étendu, ou pour la présence de coloration, et les homozygotes pour la crête simple, ont un âge à maturité sexuelle moins variable, respectivement, que les poules à noir restreint, à "blanc récessif $*$ ou à crête en rose. Dans ces trois cas, les moyennes ne diffèrent pas, et le génotype ayant la plus grande variance phénotypique s'accompagne d'une composante " père "de la variance proportionnellement plus élevée.

Aux loci $R$ et $C$, la comparaison des croisements réciproques suggère un effet maternel sur la variance de l'âge au 1 er ouf.

\section{INTRODUCTION}

Dans les effets quantitatifs associés à un gène donné, l'on ne mentionne très généralement que son influence sur la valeur moyenne d'un caractère. Or, il n'est pas inconcevable qu'un locus simple, ou une région chromosomique d'étendue limitée, puisse avoir une action sur d'autres paramètres biométriques ou génétiques telles la variance phénotypique, sa composition, ou encore des corrélations entre caractères.

Des exemples illustrant ces possibilités sont rares; à vrai dire, ils ont été peu recherchés. Un fait au moins a été mis en évidence et soumis à de nombreuses 
vérifications expérimentales : il s'agit de la variabilité attribuable à des causes non génétiques, souvent plus grande chez des animaux consanguins, comparés aux individus plus hétérozygotes. LERNER (I954) a rassemblé des résultats assez divers à l'appui de cette opinion, suivi par d'autres chercheurs.

De même, la description de mutants " anormaux " ou létaux donne souvent l'impression d'une variabilité accrue de certains aspects du développement; ce dernier est "canalisé " de façon imparfaite, suivant la terminologie adoptée par WADDINGTON (I957).

Par contre, il semble que l'on se soit préoccupé très rarement, en particulier chez les espèces d'intérêt économique, de l'effet sur la variance de gènes que 1'on peut appeler " normaux ".

Chez la Poule domestique, divers auteurs, à partir de populations indépendantes, ont noté que les coquelets hétérozygotes $\mathrm{K} k$ pour le gène lié au sexe responsable de la rapidité d'emplumement ont un poids significativement plus variable que les homozygotes $k k$ vers 1'âge de 8-Io semaines, sans que ceci s'accompagne d'une différence importante des valeurs moyennes (HuRRy et NordskoG, r953; GlazenER et JULL, I946; GODFREy et FARNSWORTH, I952; GOODMAN et MUIR, I965; MÉRAT, I967a).

Un résultat analogue est suggéré par Goodman et MuIR (I965) pour le locus $\mathrm{P}$ (crête en pois/crête simple) et par CROBER et HAwEs (I966) pour le gène $R$ (crête en rose/crête simple). Nous avons d'autre part signalé que l'extension du noir de type "sauvage " pour le plumage, attribuable sans doute en majeure partie à un gène simple, s'accompagnait d'une variance réduite du poids à 8 semaines (MÉRAT, I958), et que le taux de fertilité des mâles porteurs du gène "lisse " modifiant la texture des crêtes en rose était à la fois meilleur en moyenne et plus homogène que celui des porteurs de 1'allèle " hérissé " (CAVALIE et MÉRAT, I965).

Nous donnerons ici d'autres exemples de loci associés à un effet relativement important sur la variance d'un caractère ou la composition de celle-ci; ils se rapportent à 1'âge de la maturité sexuelle des poulettes, estimé d'après la ponte du premier œuf.

\section{MATÉRIEL, ET MÉTHODES}

Dans la population étudiée, issue d'un croisement de plusieurs races fait en 1954 (MÉRAT, $1962,1967 a$ ), divers loci sont maintenus en ségrégation. C'est le cas pour C (présence /absence de mélanines dans le plumage), $\mathbf{E}$ (Extension du noir dans le plumage /noir restreint), $\mathbf{R}$ (crête en rose /crête simple). Ces gènes ont été décrits par HuTt (1949). La classification des génotypes ne présente pas de difficultés, sauf parfois pour l'extension du noir, où le classement en deux catégories comporte un certain pourcentage de cas douteux, et où l'expression du gène principal en cause diffère suivant le sexe à l'état hétérozygote. De toute facon, nous ne prenons ici en considération que la différence entre noir complètement ou incomplètement étendu, le premier caractère pouvant raisonnablement être associé à un allèle dénommé $E$ d'un locus présentant sans doute toute une série d'allèles agissant en interaction avec d'autres loci (cf. par exemple Kimball, 1952; Brumbaugh et Hollander, 1963; Smyth, 1965).

Cette population est reproduite en évitant toute consanguinité systématique. Elle a été subdivisée à l'origine en deux troupeaux séparés, élevés l'un au printemps, l'autre à l'automne. Pour chacun, le nombre de reproducteurs par génération est voisin de 20 đo et 160 ợ. Tous les accouplements réalisés sont pedigree avec un seul coq accouplé à une poule déterminée et 8 à 10 poules par coq. L'élevage est fait au sol, pour les jeunes dont la croissance est enregistrée, puis pour les poulettes dont la ponte individuelle est contrôlée; les animaux des divers génotypes et familles sont élevés dans les mêmes locaux. 
Pour chaque locus, nous avons cherché les différences quantitatives pouvant lui être associées dans les familles de sceurs dont un parent était hétérozygote et l'autre homozygote récessif. La comparaison des moyennes des deux génotypes était faite sur des couples de sœurs nées le même jour et élevées ensemble. C'est à partir de ces mêmes couples que la variance phénotypique a été comparée. Une étude plus complète portant sur tous les descendants des familles présentant une ségrégation aux loci en cause était refaite ensuite dans le cas du facteur "extension du noir " pour lequel les effectifs étaient moins nombreux.

Parmi les critères quantitatifs dont la variance était enregistrée, l'âge à la ponte du premier œuf des poulettes a donné des différences hautement significatives : comme nous l'avons dit plus haut, c'est lui, essentiellement, qui sera considéré ici. Il s'agit du premier œuf contrôlé au nid-trappe, sujet à erreurs du fait d'un certain pourcentage de pontes non enregistrées, de l'ordre de 1 à 3 p. 100 suivant l'année. Il est vraisemblable que la fréquence des erreurs ne diffère pas pour les génotypes comparés : si c'était le cas, les âges moyens observés devraient différer.

Pour chaque locus, nous ne mentionnerons que les résultats globaux, sur l'ensemble des générations étudiées, n'ayant pas constaté de variations significatives du sens de ces résultats suivant l'année.

Du point de vue statistique, pour les tests de comparaison de variances, nous avons dû dans quelques cas suppléer à l'imprécision des tables existantes, pour les grands nombres de degrés de liberté, en utilisant le fait que la distribution de $\frac{\log F}{2}$ tend alors vers une distribution normale dont les moments sont donnés par Kendall et Stuart (1963).

\section{RÉSULTATS}

\section{a) Noir ètendu ou restreint dans le plumage}

Sur I68 couples de sœurs nées le même jour, l'une à plumage noir étendu hétérozygote pour l'allèle E (HUTT, I949), 1'autre à noir restreint, ne possédant pas $\mathrm{E}$, la moyenne de l'âge au premier œuf et sa variance intra-année ont été comparées (tabl. I). Les résultats groupent 9 années du troupeau reproduit à l'automne, de I956 à rg64.

\section{TABLEAU I}

Moyenne et variance de l'âge au premier aut, poulettes à noir étendu ou restreint

\begin{tabular}{|c|c|c|c|c|c|c|}
\hline \multirow{2}{*}{ Phénotype } & \multirow{2}{*}{$\begin{array}{c}\text { Nombre } \\
\text { d'individus }\end{array}$} & \multirow{2}{*}{$\begin{array}{l}\text { Age } \\
\text { moyen } \\
\text { (jours) }\end{array}$} & \multirow{2}{*}{$\begin{array}{l}\text { Compa- } \\
\text { raison } \\
\text { des } \\
\text { moyennes }\end{array}$} & \multicolumn{2}{|c|}{$\begin{array}{l}\text { Variance } \\
\text { intra-année }\end{array}$} & \multirow{2}{*}{$\begin{array}{l}\text { Rapport } \\
\text { des } \\
\text { variances } \\
\text { (F) }\end{array}$} \\
\hline & & & & $\begin{array}{c}\text { Degrés } \\
\text { de liberté }\end{array}$ & $S^{2}$ & \\
\hline Noir étendu & 168 & 163,8 & $t=0,276$ & I 59 & 263,8 & $F=2,00$ \\
\hline Noir restreint & I 68 & 163,2 & N.S. & 159 & $5 \geq 4,6$ & \\
\hline
\end{tabular}

Alors que les moyennes sont pratiquement identiques, les variances diffèrent de façon hautement significative, celle des poules à noir limité (" non $\mathrm{E}$ ") étant double de l'autre.

Une certaine proportion d'entrées en ponte spécialement tardives, qui existe dans tous les cas, entraîne une légère dissymétrie des distributions. Celle-ci n'était pas assez importante pour perturber de façon notable les tests de comparaisons de 
moyennes, dont la " robustesse " est assez grande. Quant aux variances, étant donné le degré élevé de signification constaté, le sens de la conclusion ne peut être essentiellement modifié, quoique la probabilité indiquée puisse être quelque peu inexacte (Box, I953). C'est pourquoi nous n'avons pas jugé nécessaire d'effectuer une transformation des données, transformation dont le choix aurait été difficile.

D'autre part, la similitude des moyennes suggère que la différence des variances ne s'explique pas uniquement par une différence dans la proportion d'entrées en ponte très tardives. L'examen, année par année, des histogrammes de fréquences des âges à maturité sexuelle le confirme : il y a régulièrement un étalement plus grand des entrées en ponte chez les poules à pigmentation réduite, aussi bien vers la gauche que vers la droite de la distribution. On le vérifie globalement en transformant les données brutes en écarts aux moyennes annuelles, et réunissant toutes les années en une distribution unique pour chacun des 2 groupes comparés. Par exemple, le pourcentage des poules d'au moins 30 jours plus précoces que la moyenne annuelle est, chez les " $\mathrm{E}$ ", de 2,34, contre 4,63 chez les " non $\mathrm{E}$ "; les valeurs correspondantes pour les individus d'au moins 30 jours plus tardifs que la moyenne sont respectivement 7,33 et 9,57 p. Ioo. Pour les poules de 40 jours au moins plus tardives que la moyenne, on trouve 4,69 et $7,18 \mathrm{p}$. Ioo. Un test de $\chi^{2}$ permet de vérifier que ces proportions sont, à chaque fois, significativement différentes 1'une de l'autre.

$\mathrm{Au}$ vu de ce résultat, nous avons analysé, pour chacun des 2 groupes, la différence globale de variance en fonction de 2 facteurs : la date d'éclosion d'une part, la famille paternelle de l'autre. Pour cette analyse, nous avons repris, dans chaque année, toutes les poules à entrée en ponte contrôlée, issues des familles présentant une ségrégation pour le facteur de coloration étudié.

Le tableau 2 donne le détail de la variance, pour les poules " $E$ " ou " non $E$ ", intra- et entre dates d'éclosion intra-année, puis entre années, ainsi que les rapports $\mathrm{F}$ des 2 dernières variances à la première.

TABLEAU 2

Variance intra- et entre dates d'éclosion et années, poules à noir étendu ou non

\begin{tabular}{|c|c|c|c|c|c|c|c|c|}
\hline \multirow{2}{*}{$\begin{array}{ll} & \text { Source } \\
\text { de } & \text { variation }\end{array}$} & \multicolumn{4}{|c|}{ Noir étendu } & \multicolumn{4}{|c|}{ Noir restreint } \\
\hline & $\begin{array}{l}\text { Degrés } \\
\text { de } \\
\text { liberté }\end{array}$ & Variance & $\mathrm{F}$ & $\mathbf{P}$ & $\begin{array}{c}\text { Degrés } \\
\text { de } \\
\text { liberté }\end{array}$ & Variance & $\mathrm{F}$ & $\mathbf{P}$ \\
\hline $\begin{array}{l}\text { Entre années. } \\
\text { Entre dates d'é- } \\
\text { closion intra- } \\
\text { année... . } \\
\text { Résiduelle.. . }\end{array}$ & $\begin{array}{r}23 \\
307\end{array}$ & $\begin{array}{l}421,4 \\
355,6\end{array}$ & 24,70 & $<0,001$ & $\begin{array}{r}24 \\
546\end{array}$ & $\begin{array}{r}1032,8 \\
546,0\end{array}$ & 26,86 & $\begin{array}{l}<0, \mathrm{OOI} \\
\simeq \mathrm{o,or}\end{array}$ \\
\hline
\end{tabular}


Pour chacune des 3 variances (résiduelle, entre dates d'éclosion, entre années), on retrouve une valeur plus faible pour le groupe à noir étendu que pour l'autre. Cependant, il est intéressant de noter que la variance entre dates d'éclosion est proportionnellement plus augmentée lorsqu'on passe du groupe "noir étenđu " à celui à " noir restreint "; c'est ce que reflète d'une autre façon la comparaison du rapport $\mathbf{F}$ de cette variance à la variance résiduelle, rapport légèrement différent de I, mais non significativement, pour le premier groupe, supérieur à I au seuil de probabilité I p. Ioo pour le second. Autrement dit, la variance plus grande de la date d'entrée en ponte des poules à pigmentation restreinte peut s'expliquer, au moins partiellement, par une sensibilité accrue à des facteurs externes, associés en particulier à la date de naissance.

L,e tableau 3 indique, de même, le détail de la variance intra-pères et entre pères intra-années.

TABI EAU 3

T'ariance intra-et entre familles de père, poules à noir étendu ou non

\begin{tabular}{|c|c|c|c|c|c|c|c|c|}
\hline \multirow{2}{*}{ de $\begin{array}{l}\text { Source } \\
\text { variation }\end{array}$} & \multicolumn{4}{|c|}{ Noir étendu } & \multicolumn{4}{|c|}{ Noir restreint } \\
\hline & $\begin{array}{c}\text { Degrés } \\
\text { de } \\
\text { liberté }\end{array}$ & Variance & $F$ & $\mathrm{P}$ & $\begin{array}{c}\text { Degrés } \\
\text { de } \\
\text { liberté }\end{array}$ & Variance & $F$ & $\mathbf{P}$ \\
\hline $\begin{array}{r}\text { Entre pères in- } \\
\text { tra-années . } \\
\text { Intra-pères. }\end{array}$ & $\begin{array}{r}34 \\
277\end{array}$ & $\begin{array}{l}523,5 \\
344,8\end{array}$ & $I, 52$ & $\simeq 0,05$ & $\begin{array}{r}34 \\
468\end{array}$ & $\begin{array}{r}982,7 \\
545,2\end{array}$ & 3,64 & $<\mathrm{O}, \mathrm{OI}$ \\
\hline
\end{tabular}

Ici encore, tant la variance intra-pères que celle entre pères est indiscutablement inférieure chez les poules noires, mais la seconde est proportionnellement beaucoup plus augmentée que la première lorsqu'on passe du phénotype " noir étendu " au " noir restreint". Si l'on compare les 2 variances " entre pères " et " intra-pères" dans chaque classe phénotypique, le rapport $F$ passe de $I, 52$ (à la limite de la signification) pour les poules " $\mathrm{E}$ " à 3,64 (hautement significatif) pour les " non $E$ ". Ou, pour voir les choses d'une autre manière, le rapport $F$ de la variance résiduelle chez les " non $\mathrm{E}$ " à celle des " $\mathrm{E}$ " est 1,58 (avec 277 et 468 degrés de liberté), le rapport analogue relatif aux variances entre pères étant 3,78 (avec 34 et 34 d.1.).

On peut tester d'une façon approximative la différence de ces 2 derniers rapports $\mathrm{F}$. Si l'on prend comme "vraie valeur " du rapport des variances des " non $\mathrm{E}$ " aux " $\mathrm{E}$ " le rapport observé des variances résiduelles égal à $\mathrm{I}, 58$, en appelant respectivement $S_{1}^{2}$ et $S_{2}^{2}$ les variances observées " entre pères ", le rapport $\frac{S_{2}^{2}}{1,5^{8} S_{1}^{2}}$ devrait suivre approximativement une loi de $\mathrm{F}$ dans l'hypothèse d'un rapport égal pour toutes les composantes de la variance lorsqu'on passe du groupe " noir restreint " au groupe " noir étendu ". Or, sa valeur calculée à partir des observations 
est 2,39, significative au seuil I p. Ioo, et il en est encore de même si, au lieu de $x, 58$, on prend l'une des limites de confiance au seuil $5 \mathrm{p}$. Ioo du "vrai " rapport des variances résiduelles.

Si l'on estime maintenant, dans les données précédentes, la composante " père " de la variance ( ${ }^{1}$ ) chez les poules " $\mathrm{E}$ " et " non $\mathrm{E}$ ", on trouve les valeurs respectives $22, \mathrm{I}$ et $\mathrm{I} 08,8$.

Ces résultats permettent donc de conclure que la présence du gène $\mathrm{E}$ opposé à ses allèles a pour effet, non seulement de diminuer globalement la variance phénotypique, mais aussi d'en modifier la composition, en diminuant la part due à certains facteurs au moins de l'environnement, ainsi que celle attribuable à des différences génétiques entre familles de pères (les effectifs très restreints par mères permettaient difficilement une analyse similaire sur ces dernières).

Enfin, le tableau 4 donne la comparaison, à partir de couples de scurs nées le même jour, des moyennes et des variances des poulettes " $E$ " ou " non $E$ " pour le nombre d'œufs pondus de l'entrée en ponte à l'arrêt du contrôle, correspondant à un âge de 9 à Io mois.

\section{TABLEAU 4}

Moyennes et variances du nombre d'aufs (ponte des premiers mois), poulettes à noir étendu ou restreint

\begin{tabular}{|c|c|c|c|c|c|c|c|}
\hline \multirow{2}{*}{ Groupe } & \multirow{2}{*}{$\begin{array}{l}\text { Nombre } \\
\text { d'indi- } \\
\text { vidus }\end{array}$} & \multirow{2}{*}{$\begin{array}{l}\text { Valeur } \\
\text { moyenne }\end{array}$} & \multirow{2}{*}{$\begin{array}{l}\text { Compa- } \\
\text { raison } \\
\text { des } \\
\text { moyennes }\end{array}$} & \multicolumn{2}{|c|}{$\begin{array}{c}\text { Variance } \\
\text { intra-années }\end{array}$} & \multirow{2}{*}{$\begin{array}{c}\text { Rapport } \\
\text { F } \\
\text { des } \\
\text { variances }\end{array}$} & \multirow{2}{*}{$\begin{array}{l}\text { Signifi- } \\
\text { cation } \\
\text { de F }\end{array}$} \\
\hline & & & & $\begin{array}{c}\text { Degrés } \\
\text { de liberté }\end{array}$ & $S^{2}$ & & \\
\hline $\begin{array}{l}\text { Noir étendu. . } \\
\text { Noir restreint. . }\end{array}$ & $\begin{array}{l}163 \\
163\end{array}$ & $\begin{array}{l}41,95 \\
44,5 \mathrm{I}\end{array}$ & $\begin{array}{l}t=\mathrm{I}, 34 \\
\text { N.S. }\end{array}$ & $\begin{array}{l}\text { I } 54 \\
\text { I } 54\end{array}$ & $\begin{array}{l}335,7 \\
499,1\end{array}$ & $I, 49$ & $\mathbf{P} \simeq 0,05$ \\
\hline
\end{tabular}

Il semble y avoir, ici aussi, une variance plus grande pour les poules à noir limité. Cette tendance, moins accusée que pour 1'âge à maturité sexuelle, peut probablement s'expliquer entièrement par la corrélation existant entre ce caractère et la ponte des premiers mois.

b) Présence ou absence de pigmentation dans le plumage (Cc/cc).

Le tableau 5 précise la valeur moyenne et la variance de l'âge au premier œuf pour les poules pigmentées $(\mathrm{C} c)$ ou à pigmentation inhibée $(c c)$ dans le plumage. Il s'agissait encore de couples de sœurs contemporaines, issues de l'accouplement d'un parent hétérozygote $\mathrm{C} c$ avec un récessif $c c$. Le détail est donné pour les 2 croisements réciproques. Les générations étudiées se situent, comme pour l'extension du noir, entre I956 et I964, mais la majorité des données est, cette fois, tirée du troupeau reproduit au printemps.

(1) Pour obtenir cette composante "père ", nous avons utilisé seulement les sommes de carrés " entre pères " et "intra-pères", sans considérer le détail des familles de frères-sours (peu nombreuses). Une part de la composante "mère " est donc incluse dans la variance "entre pères ". 
TABLEAU 5

Moyennes et variances de l'age au $I^{\text {er }}$ out, poulettes à plumage coloré (Cc) ou "blanc récessif " (cc)

\begin{tabular}{|c|c|c|c|c|c|c|c|c|}
\hline \multirow{2}{*}{$\begin{array}{c}\text { Type } \\
\text { d'accouplement } \\
\text { parental }\end{array}$} & \multirow{2}{*}{$\begin{array}{l}\text { Géno- } \\
\text { type } \\
\text { des } \\
\text { filles } \\
\text { com- } \\
\text { parées }\end{array}$} & \multirow{2}{*}{ Fffectif } & \multirow{2}{*}{$\begin{array}{c}\text { Valeur } \\
\text { moyen- } \\
\text { ne } \\
\text { (jours) }\end{array}$} & \multirow{2}{*}{$\begin{array}{l}\text { Compa- } \\
\text { raison } \\
\text { des } \\
\text { moyen- } \\
\text { nes }\end{array}$} & \multicolumn{2}{|c|}{$\begin{array}{l}\text { Variance } \\
\text { intra-année }\end{array}$} & \multirow{2}{*}{$\begin{array}{c}F \\
\text { Rapport } \\
\text { de la } \\
\text { varian- } \\
\text { ce des } \\
c c \\
\text { aux } \mathrm{C} c\end{array}$} & \multirow{2}{*}{$\begin{array}{l}\text { Signi- } \\
\text { fication } \\
\text { de F }\end{array}$} \\
\hline & & & & & $\begin{array}{l}\text { Degrés } \\
\text { de } \\
\text { liberté }\end{array}$ & $S^{2}$ & & \\
\hline $\mathrm{C} c \times c c$ & $\begin{array}{l}\mathrm{C} c \\
c c\end{array}$ & $\begin{array}{l}162 \\
162\end{array}$ & $\begin{array}{l}\text { I } 59,4 \\
\text { I } 59,9\end{array}$ & $\begin{array}{c}t=-0,26 \\
\text { N.S. }\end{array}$ & $\begin{array}{l}148 \\
\mathrm{I}_{4} 8\end{array}$ & $\begin{array}{l}496,6 \\
725,2\end{array}$ & $I, 46$ & $\mathbf{P}<_{0,025}$ \\
\hline$c c \times \mathrm{C} c$ & $\begin{array}{l}\mathrm{C} c \\
c c\end{array}$ & $\begin{array}{l}\text { I } 68 \\
\text { I68 }\end{array}$ & $\begin{array}{l}\text { I } 58,0 \\
\text { I } 60,1\end{array}$ & $\begin{array}{c}t=-\mathrm{0}, 97 \\
\mathrm{~N} . \mathrm{S} .\end{array}$ & $\begin{array}{l}\text { I57 } \\
\text { I57 }\end{array}$ & $\begin{array}{l}3^{63,8} \\
515,5\end{array}$ & $I, 42$ & $\mathrm{P} \underset{\mathrm{O}, 025}{<}$ \\
\hline
\end{tabular}

Alors que l'âge moyen ne diffère pas de façon sensible (un très léger avantage aux 우 colorées existe pour les 2 types de croisement, mais n'est pas significatif au total) on note, dans les 2 cas, une variance supérieure pour les filles $c c$, ceci étant significatif à chaque fois au seuil $2,5 \mathrm{p}$. Ioo. De même que pour l'extension de la coloration, l'examen des distributions de fréquence par année confirme qu'il y a étalement plus grand dans les 2 sens et non seulement vers les entrées en ponte tardives.

En regroupant les 2 croisements, on obtient, pour les filles colorées, une variance égale à 428,2 ; la valeur correspondante pour les filles $c c$ est $6 r_{7,3}$. L,es 2 variances sont basées sur 305 degrés de liberté, et leur rapport est $t=\mathrm{I}, 44$, ce qui correspond à une probabilité inférieure à I p. Ioo si l'on fait abstraction de l'hétérogénéité existant entre croisements réciproques, dont nous parlerons plus loin. De toute façon, le fait d'un rapport $F$ significatif, dans le même sens, pour les 2 types de croisement, conduit à cette même conclusion en considérant le nombre de tels événements (niveau de signification 0,025) obtenus sur 2 tirages comme suivant une loi binômiale.

Comme pour l'extension du noir, nous avons fait le détail de la variance intrapères et entre pères intra-génération pour les 2 génotypes (tabl. 6). Nous avons omis les variances entre années, dont le rapport à la variance résiduelle différait peu entre génotypes.

Il existe, ici comme pour l'extension du noir, une tendance à l'augmentation de la composante " père " de la variance chez les o우 les plus variables $(c c)$, mais elle est de toute façon beaucoup moins nette.

Quant au nombre d'œufs pondus les premiers mois, sa variance ne présente pas de différence sensible suivant le génotype au locus $C$ (le rapport $F$ de la variance intra-année des $c c$ aux $\mathrm{C} c$ étant de $I_{1}, 07$ avec $\mathrm{I}_{52}$ et $\mathrm{I}_{52}$ degrés de liberté pour le croisement $\mathrm{C} c \times c c$ et $0,9 \mathrm{I}$ avec $\mathrm{I}_{4} 6$ et I46 d.1. pour le croisement réciproque). 


\section{TABLEAU 6}

Variance intra- et entre lamilles de pères, poules $\mathrm{Cc}$ ou $\mathrm{cc}$

\begin{tabular}{|c|c|c|c|c|c|c|c|c|}
\hline \multirow{2}{*}{$\begin{array}{c}\text { Source } \\
\text { de variation }\end{array}$} & \multicolumn{4}{|c|}{$\mathrm{C} c$} & \multicolumn{4}{|c|}{$c c$} \\
\hline & $\begin{array}{l}\text { Degrés } \\
\text { de } \\
\text { liberté }\end{array}$ & Variance & $\mathbf{F}$ & $\mathrm{P}$ & $\begin{array}{l}\text { Degrés } \\
\text { de } \\
\text { liberté }\end{array}$ & Variances & $\mathbf{F}$ & $P$ \\
\hline 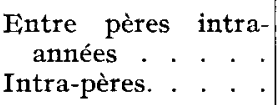 & $\begin{array}{r}3^{8} \\
257\end{array}$ & $\begin{array}{l}3^{64,2} \\
331,0\end{array}$ & $\mathrm{I}, \mathrm{IO}$ & N.S. & $\begin{array}{r}3^{8} \\
257\end{array}$ & $\begin{array}{l}560,7 \\
492,2\end{array}$ & $\mathrm{I}, 3 \mathrm{I}$ & N.S. \\
\hline
\end{tabular}

c) Crête en rose/crête simple $(R \mathrm{r} / \mathrm{rr})$.

Comme au locus $\mathrm{C}$, nous comparons moyenne et variance de la précocité sexuelle pour des couples de sœurs, l'une, cette fois de génotype, $\mathrm{R} r$, l'autre $r r$, issues d'un parent $\mathrm{R} r$ accouplé à un $r r$. Le tableau 7 contient les résultats, détaillés par croisement. Les données, comme pour $\mathrm{C} c$ et $c c$, se répartissent entre le troupeau élevé au printemps (pour la majorité) et celui reproduit en automne.

TABIEAU 7

Moyennes et variances de l'âge au I er ouf, poulettes à crête en rose $(R \mathbf{r})$ ou simple ( $\mathbf{r r}$ )

\begin{tabular}{|c|c|c|c|c|c|c|c|c|}
\hline \multirow{2}{*}{$\begin{array}{c}\text { Type } \\
\text { d'accouplement } \\
\text { parental }\end{array}$} & \multirow{2}{*}{$\begin{array}{c}\text { Géno- } \\
\text { type des } \\
\text { filles } \\
\text { com- } \\
\text { parées }\end{array}$} & \multirow{2}{*}{ Effectif } & \multirow{2}{*}{$\begin{array}{l}\text { Valeur } \\
\text { moyen- } \\
\text { ne } \\
\text { (jours) }\end{array}$} & \multirow{2}{*}{$\begin{array}{l}\text { Compa- } \\
\text { raison } \\
\text { des } \\
\text { moyen- } \\
\text { nes }\end{array}$} & \multicolumn{2}{|c|}{$\begin{array}{l}\text { Variance } \\
\text { intra-année }\end{array}$} & \multirow{2}{*}{$\begin{array}{c}F \\
\text { rapport } \\
\text { des } \\
\text { varian- } \\
\text { ces des } \\
\text { of } \mathrm{Rr} \text { et } \\
r r\end{array}$} & \multirow{2}{*}{$\begin{array}{c}\text { Signifi- } \\
\text { cation } \\
\text { de } F\end{array}$} \\
\hline & & & & & d.l. & $\mathrm{S}^{2}$ & & \\
\hline $\mathrm{R} r \times r \gamma$ & $\begin{array}{c}\mathrm{R} r \\
r r\end{array}$ & $\begin{array}{l}399 \\
399\end{array}$ & $\begin{array}{l}r 64,4 \\
\mathrm{I} 63,6\end{array}$ & $\begin{array}{c}t=+0,40 \\
\text { N.S. }\end{array}$ & $\begin{array}{l}3^{85} \\
3^{85}\end{array}$ & $\begin{array}{l}549, \mathrm{I} \\
35^{2}, 3\end{array}$ & $\mathrm{r}, 59$ & $\mathrm{P}<\mathrm{O}, \mathrm{O}$ I \\
\hline$r v \times \mathrm{R} r$ & $\begin{array}{r}\mathrm{R} r \\
r r\end{array}$ & $\begin{array}{l}329 \\
329\end{array}$ & $\begin{array}{l}\text { I } 71,4 \\
\text { I } 70,2\end{array}$ & $\begin{array}{c}t=+0,66 \\
\text { N.S. }\end{array}$ & $\begin{array}{l}316 \\
316\end{array}$ & $\begin{array}{l}793,4 \\
613,5\end{array}$ & $\mathrm{r}, 29$ & $P<0,05$ \\
\hline
\end{tabular}

Ici, encore, il n'y a pas de différence sensible des moyennes.

Pour les 2 types d'accouplement parental examinés, la variance intra-années est significativement supérieure pour les filles à crête en rose. 
Si l'on regroupe les 2 croisements réciproques, le rapport de la variance des $\mathrm{R} r$ à celle des $r r$ est égal à $\mathrm{I}, 4^{\circ}$ pour $70 \mathrm{I}$ et $70 \mathrm{O}$ degrés de liberté. Ce rapport est d'un ordre de grandeur qui serait significatif au-delà du seuil I p. Ioo et la complication introduite par l'inégalité de variance entre croisements réciproques (cf. $\S d$ ), comme pour le locus $\mathrm{C}$, ne peut modifier beaucoup le sens de cette conclusion.

Enfin, les variances intra-génération, intra-pères et entre pères, ont été estimées pour chaque génotype, à partir des individus du tableau 7 , en groupant les croisements réciproques (tab1. 8). Les variances entre années sont omises comme au locus $\mathrm{C}$, leur rapport à la résiduelle étant voisin pour les 2 génotypes.

TABLEAU 8

Variance intra- et entre familles de père, poules $R \mathrm{r}$ ou $\mathrm{rr}$

\begin{tabular}{|c|c|c|c|c|c|c|c|c|}
\hline \multirow{2}{*}{$\begin{array}{c}\text { Source } \\
\text { de variation }\end{array}$} & \multicolumn{4}{|c|}{$\mathrm{R} r$} & \multicolumn{4}{|c|}{$r r$} \\
\hline & $\begin{array}{c}\text { Degrés } \\
\text { de liberté }\end{array}$ & Variance & $\mathbf{F}$ & $\mathbf{P}$ & $\begin{array}{c}\text { Degrés } \\
\text { de liberté }\end{array}$ & Variance & F & $\mathbf{P}$ \\
\hline $\begin{array}{l}\text { Entre pères, } \\
\text { intra-années. . . } \\
\text { Intra-pères . . . . }\end{array}$ & $\begin{array}{r}77 \\
6 \mathrm{I} 6\end{array}$ & $\begin{array}{r}\text { I } 78,3 \\
563,1\end{array}$ & 3,05 & $<$ & $\begin{array}{r}77 \\
616\end{array}$ & $\begin{array}{l}755,9 \\
466,4\end{array}$ & 1,62 & $<\mathrm{O}, \mathrm{OI}$ \\
\hline
\end{tabular}

La même tendance se fait jour que pour les deux autres gènes : le génotype le plus variable $(\mathrm{R} r)$ est en même temps celui pour lequel la variance " entre pères " est la plus grande vis-à-vis de la variance résiduelle, ou, en d'autres termes, celui chez lequel la composante " père " de la variance est proportionnellement la plus élevée.

Un test approximatif de la différence des rapports $F$ caractérisant les 2 génotypes, analogue à celui réalisé pour le facteur " extension du noir ", conduit à conclure à une différence hautement significative de ces rapports.

La composante " père " de la variance $\left({ }^{1}\right)$, estimée à partir des données cidessus, a pour valeur $\mathrm{I}_{4} 8,7$ et 37,3 respectivement chez les poulettes $\mathrm{R} r$ et $r r$.

Pour les nombres d'œufs pondus durant les premiers mois de production, leur moyenne ne diffère pas sensiblement et leur variance est voisine et non significativement différente pour les poules $\mathrm{R} r$ et $r$.

\section{d) Possibilités d'effets maternels.}

Nous avons séparé au tableau 7 les 2 croisements réciproques relatifs au gène $R$. Il apparaît à première vue, en effet, que leurs variances pour l'âge au premier ouf diffèrent, tant pour les filles $\mathrm{R} r$ que pour les $r r$.

(1) Contenant, comme pour le locus $\mathrm{F}$, une fraction de la composante "mère ". 
De façon plus précise, en ne gardant que les années où les 2 croisements sont représentés, les variances intra-années sur l'ensemble des données, par croisement. et génotype des filles, sont indiquées au tableau 9.

TABLEAU 9

Comparaison des croisements réciproques $R \mathrm{r} \times \mathrm{rr}$ et $\mathrm{rr} \times R \mathrm{r}$ pour la variance de l'age au I $^{\text {er }}$ ceuf

\begin{tabular}{|c|c|c|c|c|c|c|}
\hline \multirow{3}{*}{ Croisement } & \multicolumn{6}{|c|}{ Variance intra-année sur l'ensemble des années } \\
\hline & \multicolumn{2}{|c|}{ Filles $\mathrm{R} r$} & \multicolumn{2}{|c|}{ Filles $r \gamma$} & \multicolumn{2}{|c|}{ Ensemble } \\
\hline & D.L. & Variance & D.I. & Variance & D.L. & Variance \\
\hline $\begin{array}{l}\mathrm{R} r \times r r \\
r v \times \mathrm{R} r\end{array}$ & $\begin{array}{l}333 \\
3^{1} 1\end{array}$ & $\begin{array}{l}534,7 \\
721, \mathrm{I}\end{array}$ & $\begin{array}{l}333 \\
3 \text { I I }\end{array}$ & $\begin{array}{l}35^{2,2} \\
5^{25,7}\end{array}$ & $\begin{array}{l}666 \\
622\end{array}$ & $\begin{array}{l}443,4 \\
623,4\end{array}$ \\
\hline
\end{tabular}

Le rapport $\mathrm{F}$ de la variance du second croisement à celle du premier est respectivement égal à $\mathrm{I}, 35$ et à $\mathrm{I}, 49$ suivant que l'on considère à part les filles $\mathrm{R} r$ ou les filles $r r$. Le premier rapport est significatif au seuil $5 \mathrm{p}$. Ioo et le second au seuil I p. roo. Au total, la différence entre croisements réciproques paraît donc réelle.

Il est intéressant de remarquer qu'au locus $C$ pour lequel nous avons observé des différences "individuelles " dans la variabilité de l'âge à maturité sexuelle, il y a également une présomption de différence entre les croisements réciproques $\mathrm{C} c \times c c$ et $c c \times \mathrm{C} c:$ le rapport de la variance du premier au second est proche de $\mathrm{I}, 37$ si 1'on considère les filles de génotype $\mathrm{C} c$, et de $\mathrm{I}, 40$ pour les filles $c c$, ordres de grandeur voisins de ceux relatifs au locus $\mathrm{R}$ (ces rapports étant tous deux significatifs au seuil $5 \%$ ).

Pour l'extension du noir (E), les données étaient insuffisantes pour comparer des croisements réciproques, peu d'années comportant les 2 à la fois avec des effectifs suffisants. Toutefois, à première vue, il ne semble pas $\mathrm{y}$ avoir de différence nette des variances suivant le génotype maternel.

Sans que l'on puisse avancer une hypothèse sur la cause de ces différences entre accouplements réciproques, il paraît plausible de les attribuer à un effet maternel.

Nous omettons le détail de la variance intra-pères et entre-pères pour chaque croisement réciproque au locus $C$, les effectifs étant assez faibles si 1'on se limite aux années présentant à la fois les 2 croisements.

Pour le gène $\mathrm{R}$, on retrouve, pour les types d'accouplement $\delta \mathrm{R} r \times$ 우 $r r$ et o $r r \times$ 우 $\mathrm{R} r$, un fait déjà observé dans les comparaisons entre génotypes " individuels " à chaque locus : le croisement le plus dispersé au total est aussi celui où la variance " entre pères " est, à proportion, la plus grande, spécialement chez les enfants $\mathrm{R} r$, les plus variables (tab1. Io). 
TABLEAU IO

Variances "entre pères " et "intra-pères " pour les croisements réciproques au locus $R$

\begin{tabular}{|c|c|c|c|c|c|c|c|c|}
\hline \multirow{3}{*}{$\begin{array}{l}\text { Source } \\
\text { de variation }\end{array}$} & \multicolumn{4}{|c|}{$\delta \mathrm{R} r \times \stackrel{9}{r} r$} & \multicolumn{4}{|c|}{$\sigma r r \times+\mathrm{R} r$} \\
\hline & \multicolumn{2}{|c|}{ Enfants $\mathrm{R} r$} & \multicolumn{2}{|c|}{ Enfants $\gamma r$} & \multicolumn{2}{|c|}{ Enfants $\mathrm{R} r$} & \multicolumn{2}{|c|}{ Enfants $r r$} \\
\hline & d.l. & $s^{2}$ & d.l. & $s^{2}$ & d.l. & $s^{2}$ & d.l. & $s^{8}$ \\
\hline \multirow[t]{2}{*}{$\begin{array}{l}\text { Entre pères, } \\
\text { (Intra-années) } \\
\text { Résiduelle. . }\end{array}$} & $\begin{array}{r}35 \\
33^{2}\end{array}$ & $\begin{array}{l}890,5 \\
496,6\end{array}$ & $\begin{array}{r}35 \\
332\end{array}$ & $\begin{array}{l}473,2 \\
370,8\end{array}$ & $\begin{array}{r}31 \\
27^{\circ}\end{array}$ & $\begin{array}{r}2366, \mathrm{I} \\
663,5\end{array}$ & $\begin{array}{r}31 \\
270\end{array}$ & $\begin{array}{l}743,2 \\
55^{2}, 5\end{array}$ \\
\hline & \multicolumn{2}{|c|}{$\begin{array}{l}\mathrm{F}=\mathrm{I}, 79 \\
\mathrm{P}<0, \mathrm{OI}\end{array}$} & \multicolumn{2}{|c|}{$\begin{array}{c}F=I, 28 \\
\text { N.S. }\end{array}$} & \multicolumn{2}{|c|}{$\begin{array}{l}\mathbf{F}=3,57 \\
\mathrm{P}<0, \text { OOI }\end{array}$} & \multicolumn{2}{|c|}{$\begin{array}{c}F=\mathrm{I}, 34 \\
\text { N.S. }\end{array}$} \\
\hline
\end{tabular}

e) Comparaisons relatives à d'autres gènes.

Une comparaison analogue des variances intra-années pour l'âge au premier œuf entre génotypes individuels a pu être réalisée relativement à d'autres gènes, les comparaisons étant les suivantes :

I $i$ /ii (inhibition/présence de pigment noir dans le plumage).

$\mathrm{B} l b l / b l b l$ (dilution du noir/non dilution).

S-/s- (plumage "argenté "/" doré ").

Ww/ww (absence/présence de xanthophylles épidermiques).

$\mathrm{K}$ - $k$ - (emplumement lent/rapide).

Dans tous les cas il n'y avait pas de différence significative pour la valeur moyenne de l'âge au $\mathrm{I}^{\mathrm{er}}$ œuf. Il en était de même des variances, à l'exception, peut-être, d'une différence au locus $\mathrm{K}$, significative au seuil $5 \mathrm{p}$. Ioo, mais assez faible (tabl. II).

TABLEAU I I

Comparaison des variances pour l'âge au $I^{\text {er }}$ ceuf à d'autres loci

\begin{tabular}{|c|c|c|c|c|c|}
\hline & \multicolumn{5}{|c|}{ Génotypes comparés } \\
\hline & I $i$ et $i i$ & B $l b l$ et $b l b l$ & S- et $s-$ & $\mathrm{W} w$ et $w w$ & $\mathrm{~K}-$ et $k$ - \\
\hline $\begin{array}{l}\text { Degrés de liberté (I) } \\
\text { F (rapport de la plus } \\
\text { grande à la plus pe- } \\
\text { tite variance) . . . } \\
\text { Signification... . . . }\end{array}$ & $\begin{array}{l}284 \\
\\
\text { I,I } 4 \\
\text { N.S. }\end{array}$ & $\begin{array}{l}.33 \mathrm{I} \\
\text { I,03 } \\
\text { N.S. }\end{array}$ & $\begin{array}{l}600 \\
\\
\text { I,04 } \\
\text { N.S. }\end{array}$ & $\begin{array}{l}869 \\
\\
\text { I,I } 3 \\
\text { N.S. }\end{array}$ & $\begin{array}{c}77^{2} \\
P<0,05\end{array}$ \\
\hline
\end{tabular}

(1) Les degrés de liberté sont les mêmes pour chacune des deux variances comparées, les données étant constituées de couples de sceurs nées à la même date. 
De façon parallèle, le tableau I 2 concerne les comparaisons entre croisements réciproques là où elles étaient possibles, toujours limitées aux variances.

TABLEAU I 2

Comparaison entre croisements réciproques à d'autres loci pour la variance intra-années de l'áge au $I^{\text {er }}$ oeut

\begin{tabular}{|c|c|c|c|}
\hline & \multicolumn{3}{|c|}{ Croisements comparés } \\
\hline & $\mathbf{I} i \times i i$ et $i i \times \mathbf{I} i$ & $\begin{array}{c}\mathrm{B} l b l \times b l b l \\
\text { et } b l b l \times \mathrm{B} l b l\end{array}$ & $\begin{array}{l}\mathrm{W} w \times w w \\
\text { et } w w \times W w\end{array}$ \\
\hline 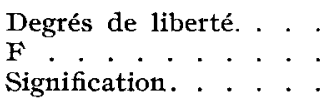 & $\begin{array}{c}254 \text { et } 34^{2} \\
\text { I,O7 } \\
\text { N.S. }\end{array}$ & $\begin{array}{c}374 \text { et } 288 \\
1,26 \\
\mathrm{P}<0,05\end{array}$ & $\begin{array}{c}978 \text { et } 760 \\
1,23 \\
P<0,05\end{array}$ \\
\hline
\end{tabular}

Dans l'ensemble, en ce qui concerne les différences de variance attachées à l'individu, 4 comparaisons sur 8 se sont donc révélées significatives, dont 3 au seuil I p. Ioo et I au seuil I p. I ooo. Or, la considération de la loi de Poisson montre que la probabilité d'obtenir du seul fait du hasard plus d'une comparaison sur Io significative au seuil I p. Ioo est inférieure à 0,0 . Un raisonnement semblable vaut pour les comparaisons entre croisements réciproques. Il est donc raisonnable de penser que les différences trouvées significatives dans des comparaisons particulières sont bien réelles.

Par contre (MÉRAT, données non publiées), nous n'avons trouvé aucune différence significative des variances, aux mêmes loci, pour les caractères suivants : poids à 8 semaines dans les 2 sexes, nombre d'œufs (sauf pour E, comme nous l'avons signalé), poids des œufs, poids des poules adultes. Seule, la variance de l'écart du poids du poussin d'un jour à sa régression sur le poids de l'œuf dont il est issu paraît, dans nos données, dépendre de certains gènes "marqueurs". Les résultats en seront publiés ultérieurement.

\section{DISCUSSION ET CONCLUSIONS}

Un premier fait, commun aux exemples qui précèdent, réside dans 1'expression de la différence de variance de l'âge au premier œuf associée à certains loci connus : les génotypes "plus variables " comportent une plus forte proportion, non seulement de " retardataires", mais aussi d'entrées en ponte précoces. Dans les 3 cas étudiés, les 2 phénomènes se compensent à peu près, de sorte que l'âge moyen ne diffère pas de façon appréciable.

LowRy et SchulTz (I959) ont suggéré la possibilité d'une différence de variance phénotypique pour un caractère quantitatif, liée à un gène marqueur, dans certains cas de linkage entre ce marqueur et un gène à effet " quantitatif ", accompagnés d'effets non additifs relativement à ce dernier gène. 
Cette explication ne paraît pas très vraisemblable dans nos exemples. Tout d'abord, les différences de variance constatées ne paraissent pas avoir diminué au cours des générations dans notre population : s'il y a un linkage, il faudrait qu'il soit assez étroit. Ensuite, comme il n'y a pas de différences appréciables des moyennes, l'effet " non additif " postulé pour le ou les gènes liés devrait être d'un type très particulier. Enfin, les différences de composition de la variance s'expliquent mal par cette hypothèse, et il y a, relativement au facteur " extension du noir ", une différence dans la variance dûe à un facteur du milieu, la date d'éclosion, qui suggère bien un effet sur la capacité de "tamponnement" ( ${ }^{1}$ ).

Nous ne disposons pas, pour l'instant, d'éléments d'interprétation du mode d'action des gènes en cause. On pourrait penser à une sensibilité plus ou moins grande à des facteurs de l'environnement capables d'influer sur la précocité sexuelle, l'expression biométrique pouvant en être une différence du coefficient de régression de l'âge à maturité sexuelle par rapport à ces facteurs.

Du point de vue physiologique, on peut se demander si 1'association entre l'extension des mélanines et le groupement des entrées en ponte dans le temps est fortuite. Le parallélisme des résultats concernant 2 gènes différents de coloration ( $\mathrm{E}$ et $\mathrm{C}$ ) pourrait, à première vue, suggérer que non. Lues observations relatives au locus I (présence/absence de mélanines noires dans le plumage) ne révèlent pas de différence significative entre génotypes à ce locus pour l'homogénéité des âges au premier ouf (tab1. Io); cependant, la variance la plus grande observée est celle des animaux $I i$ privés de noir.

Le second fait est la modification, en présence des mêmes gènes, non seulement de la variance totale, mais de sa composition. Pour les 3 loci étudiés, le génotype donnant la plus grande variance s'accompagne d'une augmentation importante de la " composante père " de celle-ci. Il est curieux de constater qu'un phénomène d'apparence analogue se révèle relativement à l'effet d'un facteur du milieu, la date d'éclosion plus ou moins tardive au printemps : chez les animaux nés tard, l'âge d'entrée en ponte est plus variable, et en même temps la composante " père " de la variance a une plus grande importance proportionnelle, manifestant peut-être l'action de gènes liés au sexe (MÉ,RAT, I956, r968).

Ainsi, tout le système de paramètres génétiques quantitatifs pour un caractère peut être modifié par un gène simple, ou au moins une région chromosomique d'étendue restreinte. C'est, si l'on veut, un cas d'épistasie, entre un gène et l'expression, non d'autres gènes spécifiés, mais du reste du génome, ou de classes entières de gènes (liés au sexe dans le cas actuel?) dans 1'hypothèse vraisemblable d'un déterminisme polygénique, mis à part le locus en question.

Il n'y a pas, à notre connaissance, d'autres cas connus d'un gène identifié modifiant à lui seul l'héritabilité d'un caractère; mais un exemple a été suggéré chez la poule d'une modification, associée au gène $\mathrm{P}$ de forme de la crète, portant sur un autre paramètre biométrique, la corrélation phénotypique entre 2 caractères quantitatifs; il s'agit de la taille de la crête d'une part, de la vitesse de croissance ou de la fertilité de 1'autre (KAN et al., r959). Nous avons nous-mêmes constaté

(1) Ces derniers faits, de même, ne semblent pas favorables à l'hypothèse d'allèles " $c$ " ou " $c$ " différant par leur effet moyen sur l'âge aul $I^{\text {er }}$ cuf mais indiscernables extérieurement.

Annales de génétique animale. - I970. 
d'autres exemples de ce genre relatifs au gène dw réduisant la taille dans la même espèce : en présence de ce gène apparaît une corrélation positive importante entre le poids adulte d'une poule et son intensité de ponte, corrélation qui n'existe pas chez des animaux de taille normale de même origine (Ḿ́rar, rg69).

Ces résultats sont intéressants pour la compréhension de l'effet d'une sélection sur un caractère métrique. Ils représentent de nouveaux exemples de gènes ayant une action notable sur la variance d'un tel caractère, et nous avons montré théoriquement les résultats particuliers auxquels une sélection soit " directionnelle ", soit "stabilisante", peut conduire pour des gènes de ce type (MÉRAT, I $967 b$ ).

On peut prévoir que ces gènes, à leur tour, auront, par la modification de la variance et de ses composantes dont ils sont responsables, un effet sur le résultat de la sélection aux autres loci. Alors qu'il est couramment reconnu (par exemple LERNER, I958) que la rapidité des gains génétiques et les limites de la sélection pour un caractère dépendent de la composition de départ d'une population, nos données montrent que l'influence de cette composition peut être, pour une part appréciable, le fait d'un petit nombre de loci particuliers. Les conséquences de cette possibilité ne semblent jamais avoir été envisagées théoriquement, mais sa prise en considération nous paraît de nature à apporter, au moins dans certains cas, un élément nouveau pour la compréhension de la génétique quantitative.

Reçu pour publication en octobre 1969.

\section{REMERCIEMENTS}

Nous remercions M. Boyer, Station de Recherches avicoles, C.R.V.Z., 37-Nouzilly, et M. Ricard, Station expérimentale d'Aviculture du Magneraud, 17-Saint-Georges-du-Bois, des critiques et remarques qu'ils nous ont apportées en relisant ce texte.

\section{SUMMARY}

\section{GENES INFLUENCING PHENOTYPIC VARIANCE OF AGE AT FIRST EGG} AND ITS COMPOSITION IN THE DOMESTIC HEN

In a " synthetic" poultry population, issue of an initial cross between breeds, the variance of several quantitative traits was compared for heterozygous vs, homozygous recessive genotypes at loci with visible effect (colour or external morphology).

A highly significant difference was found at three loci for age at first egg laying of pullets: E /other alleles (extension/limitation of black in the plumage), $\mathrm{C}$ (presence /absence of melanins in the plumage), $\mathbf{R}$ (rose/single comb). Birds heterozygous for extended black, or for presence of color (Cc), and recessive homozygotes for single comb (rr) have a less variable age, respectively, at sexual maturity than pullets with restricted black, " recessive white" plumage or rose comb. The ratio of the higher to the lower variance is 2,0 in the first case $(P<.001), 1.4$ in the second $(\mathrm{P}<.01)$, and 1.3 or 1.6 in the third, according to type of mating considered $(\mathrm{P}<.01)$. A difference of variance in the same direction for egg production during the first laying months was found for the " extension of black" gene. None of these differences is accompanied by a detectable effect on mean values, and an examination of observed distributions within years confirms that, in each case, the higher variance reflects higher frequencies at both ends of the distribution.

Moreover, in the three cases concerning age at first egg, the genotype with the higher phenotypic variance also shows a much higher " sire " variance component. 
Finally, at the two loci $\mathrm{R}$ and $\mathrm{C}$, which could be studied from this point of view, a comparison of reciprocal crosses done with one parent heterozygous and the other recessive homozygous suggests a maternal effect on variance of age at first egg in the progeny, with $\mathrm{Rr}$ or $c c$ dams having significantly more heterogeneous daughters than $r r$ or Cc ones.

These results seem to be the first to show the possibility of an important effect associated with a single locus, not only on the phenotypic variance, but on its composition, or in other words on the heritability of a trait. This may have some consequences for interpreting selection results.

\section{RÉFÉRENCES BIBIIOGRAPHIQUES}

Box G. F. P., 1958. Non-normality and tests on variances. Biometrika, 40, 318-335.

Brumbaugh J. A., Hollander W. F., 1963. A further study of the E pattern locus in the fowl. Genctics, 48,884 .

Cavalie A., Mérat P., I965. Un nouveau gène, modificateur de la forme des crêtes en rose, et son incidence possible sur la fertilité des coqs. Ann. Biol. anim. Biochim. Biophys., 5, 451-468.

Crober A. C., Hawes R. O., I966. The influence of comb types on growth rate in the domestic fowl. Brit. Poultry Sci., 7, $24 \mathrm{I}-247$.

Glazener E. W., JULL M. A., 1946. Rate of feathering and ro-week body weight observations in strains differing in shank length. Poultry Sci., 25, 433-439.

Godfrey G. F., FARnsworTy G. M., Jr, I952. Relation of the sex-linked rapid feathering gene to chick growth and mortality. Poultry Sci., 31, 65-68.

Goodman B. I., MUIR F. V., 1965. The influence of comb and feathering phenotypes on body weight and dressing percentages in broilers. Poultry Sci., 44, 644-648.

HURRY H. F., Nordskog A. W., 1953. A genetic analysis of chick feathering and its influence on growth rate. Poultry Sci., 32, $18-25$.

HuTT F. B., 1949. Genetics of the fowl. XI + 590 p. Mc Graw Hill Book Co, New York.

Kan J., Manasco B. D., Gyles N. R., Smith R. M., 1959. The effect of comb-type genotypes on six metric traits in a population of meat-type chicken. Poultry Sci., 38, 826-827.

Krndali, M. G., StuakT A., I963. The advanced theory of statistics. and ed., VIII + 433 p. Vol. I, Griffin and $\mathrm{C}^{\circ}$, London.

Kimball F., 1952. Genetic relation of extended black to wild type plumage pattern in the fowl. Poultry Sci., 31, 73-79.

LERnER I. M., 1954. Genetic homeostasis. VII + I34 p. Oliver and Boyd, I,ondon.

I,ERNer I. M., 1958. The genetic basis of selection. XVI +298 p. Wiley and Sons, New York.

Mérat P., 1956. L'effet de la date d'éclosion sur le résultat de la sélection pour la précocité sexuelle des volailles. Ann. Zootech., 5, I73-r94.

Mérat P., I958. Génétique factorielle et production chez les volailles. I. Plumage " herminé "ou " sautvage ": Relation avec la croissance en poids. Ann. Zootech., 7, 123-142.

Mérat P., 1962. Quelques relations entre caractères extérieurs à hérédité simple et productivité. C.R. $X I I^{\mathbf{e}}$ Congrès mondial d'A viculture, Syndey, $7_{1-76}$.

Mł́rat P., I967a. Contribution à l'étude de la "valeur sélective "associée à quelques gènes chez la poule domestique. I. Différences quantatitives liées au génotype individuel. A mn. Biol. anim. Biochin. Biophys., 7, 79-104.

Mírat $P .$, 1967b. I.es gènes influant sur la variance d'un caractère quantitatif et leurs répercussions possibles sur la sélection. Ann. Génét., 10, 212-220.

Múrat P., 1968. Héritabilité des caractères de ponte suivant la date d'éclosion. Ann. Zootech., 17, $467-468$.

Mérat P., 1969. Étude d'un gène de nanisme lié au sexe chez la poule. I. Description sommaire et performances. Ann. Génét. Sélec. anim., 1, 19-26.

Smyti J. R., Jr, 1965. Allelic relationship of genes determining extended black, wild type and brown plumage patterns in the fowl. Poultry Sci., 44, 89-97.

WADDINGTON C. H., 1957. The strategy of the genes. IX +262 p. Allen et Unwin, London. 